\title{
Indicadores antropométricos, composición corporal y limitaciones funcionales en ancianos
}

\author{
Patricia Arroyo, Lydia Leraa, H ugo Sánchez, \\ D aniel Bunout, José Luis Santos ${ }^{\mathrm{b}}$, Cecilia Albala.
}

\section{Anthropometry, body composition and functional limitations in the elderly}

Background: Functional limitations limit the independence and jeopardize the quality of life of elderly subjects. Aim: To assess the association between anthropometric measures and body composition with functional limitations in communityliving older people. Material and Methods: Cross-sectional survey of 377 people $\geq 65$ years old (238 women), randomly selected from the SABE/Chile project. Complete anthropometric measurements were done. Handgrip muscle strength was measured using dynamometers. Body composition was determined using Dual-Energy X-Ray Absorptiometry. Functional limitations were assessed using self reported and observed activities. Results: Body mass index was strongly associated with fat mass (men $r=0.87$; women $r=0.91$ ) and with lean mass (men $r$ $=0.55$; women $r=0.62$ ). Males had significantly greater lean mass ( $48.9 \mathrm{~kg}$ vs $34.9 \mathrm{~kg}$ ), and bone mass than females ( $2.6 \mathrm{~kg}$ vs $1.8 \mathrm{~kg}$ ) and women had higher fat mass than men $(26.3 \mathrm{~kg}$ vs $22.9 \mathrm{~kg}$ ). The prevalence of functional limitations was high, affecting more women than men (63.7\% vs $37.5 \%, p<0.01)$. Functional limitations were associated with lower handgrip strength in both sexes. In the multiple regression models, with functional limitations as dependent variable and anthropometric measures as contributing variables, only hand grip strength had a significant association (negative) with functional limitation in both genders. Age was also a significant risk factor for functional limitations among women. Conclusions: Hand grip strength was strongly and inversely associated with functional limitations. Handgrip dynamometry is an easy, cheap and low time-consuming indicator for the assessment of functional limitations and the evaluation of geriatric interventions aimed to improve functional ability (Rev Méd Chile 2007; 135: 846-54).

(Key words: Aged; Anthropometry; Body composition; Frail elderly)

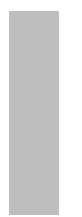

\footnotetext{
Recibido el 16 de octubre, 2006. Aceptado el 22 de enero, 2007.

Financiado por Proyecto CONICYT-MINSAL/FONIS SA04I2091.

Unidad de Salud Pública y Nutrición, Laboratorio de Epidemiología Nutricional y Genética, Instituto de Nutrición y Tecnología en Alimentos, Universidad de Chile.

aEstadístico, $\mathrm{PhD}$

bBiólogo, MSc, PhD
}

\footnotetext{
Correspondencia a: Dra. Cecilia Albala. Unidad de Salud Pública y Nutrición, Laboratorio de Epidemiología Nutricional y Genética, INTA, Universidad de Chile. Avda. El Líbano 5524, Casilla 138-11, Santiago, Chile. E mail: calbala@uchile.cl
} 
$E_{\mathrm{y}}^{1}$ acelerado proceso de transición demográfica y epidemiológica, ha originado un envejecimiento progresivo de la población y un aumento en la frecuencia de enfermedades crónicas relacionadas con la nutrición ${ }^{1}$.

El progresivo deterioro biológico y consecuente aumento de problemas de salud asociados al envejecimiento, son la resultante de la interacción de factores genéticos y ambientales, tales como estilos de vida, hábitos alimentarios, actividad física y presencia de enfermedades. La nutrición tiene un papel relevante como modulador de los cambios que provoca el envejecimiento en diferentes órganos y funciones del organismo ${ }^{2,3}$.

Las limitaciones funcionales, definidas como la restricción de las capacidades físicas o mentales para efectuar las tareas que requiere una vida independiente, son importantes predictores de mortalidad, morbilidad y discapacidad en el adulto mayor ${ }^{4,5}$. Uno de los hechos centrales que acompañan al envejecimiento son los cambios en la composición corporal ${ }^{6}$. La reducción progresiva de la masa muscular asociada al envejecimiento, llamada sarcopenia ${ }^{7}$, es un proceso natural y universal que contribuye al desarrollo de limitaciones funcionales y discapacidad en el envejecimiento ${ }^{8-10}$. Igualmente, la disminución de la fuerza muscular impacta negativamente sobre el desempeño físico y limita la movilidad, lo que se asocia con dependencia funcional y aumento de morbilidad y mortalidad ${ }^{11}$. Varios estudios epidemiológicos han mostrado asociación directa entre índice de masa corporal (IMC) y limitaciones funcionales, reportando que pesos corporales mayores se asocian con pérdida de movilidad y con limitaciones funcionales, así como también que el bajo peso se asocia a mayor riesgo de pérdida de la autonomía ${ }^{12}$.
Por otra parte, se reconoce la eficacia del ejercicio físico para disminuir la pérdida de masa magra, mejorar la fuerza muscular y la capacidad de marcha ${ }^{13}$. Ello pone de manifiesto la necesidad de contar con instrumentos de evaluación para el nivel poblacional que cumplan con criterios de validez, aplicabilidad y bajo costo. El objetivo de este estudio es determinar la asociación de indicadores antropométricos y de composición corporal con funcionalidad en el adulto mayor.

\section{MATERIAL Y MÉTODOS}

Estudio de corte transversal efectuado en 377 sujetos de 65 años, que constituyen una submuestra de los adultos mayores estudiados en el proyecto multicéntrico SABE (Encuesta Salud, Bienestar y Envejecimiento) Chile, que incluye 1.306 personas de 60 años y más, seleccionados en una muestra probabilística y representativa del Gran Santiago ${ }^{14}$. Dicha submuestra, estratificada por sexo y edad (Tabla 1), fue seleccionada de la muestra total de SABE en forma aleatoria e independiente con respecto a las características clínicas. Todos los participantes firmaron un consentimiento informado después de recibir una descripción verbal y escrita del estudio, cuyo protocolo fue aprobado por el Comité de Ética del INTA y luego fueron entrevistados para obtener datos demográficos, antecedentes de enfermedades crónicas e información sobre funcionalidad y movilidad. Luego se realizó una evaluación antropométrica completa y se les solicitó efectuar algunas acciones de movilidad.

Las capacidades funcionales se midieron usando tres tipos de variables autorreportadas: limitaciones en actividades básicas de la vida diaria

Tabla 1. C omposición de la muestra en estudio por sexo y grupo de edad

\begin{tabular}{|c|c|c|c|c|}
\hline Grupos de edad & $\begin{array}{c}\text { Hombres } \\
\mathrm{n} \quad(\%)\end{array}$ & $\begin{array}{c}\text { Mujeres } \\
\text { n (\%) }\end{array}$ & & $\begin{array}{l}\text { tal } \\
(\%)\end{array}$ \\
\hline $65-69$ & $38 \quad(27,4)$ & $68 \quad(28,6)$ & 106 & $(28,1)$ \\
\hline $70-74$ & $41 \quad(29,5)$ & $65 \quad(27,3)$ & 106 & $(28,1)$ \\
\hline $75-79$ & $32 \quad(23,0)$ & $54 \quad(22,7)$ & 86 & $(22,8)$ \\
\hline$\geq 80$ & $28 \quad(20,1)$ & $51 \quad(21,4)$ & 79 & $(21,0)$ \\
\hline Total & 139 (100) & $238 \quad(100)$ & 377 & (100) \\
\hline
\end{tabular}


(AVD), limitaciones en actividades instrumentales de la vida diaria (AIVD) y limitaciones en movilidad 0 actividades avanzadas de la vida diaria $(\mathrm{AAVD})^{15}$.

Para definir limitación funcional se utilizó el reporte de dificultades en al menos 1 AVD 01 AIVD o 2 AAVD. Este criterio desarrollado por Albala et $\mathrm{al}^{16}$, permite captar un amplio rango de limitación funcional, desde limitación leve o spreclínica», hasta limitación franca en AVD. Las variables de AVD incluidas fueron limitación en la capacidad de comer, de transferirse desde la silla a la cama y viceversa, de caminar dentro de una pieza, de vestirse, de bañarse y de usar el excusado. Las variables de AIVD estudiadas fueron limitación para realizar quehaceres livianos del hogar, realizar quehaceres pesados del hogar, lavar, cocinar, efectuar compras de almacén y alimentos, manejo de dinero, tomar sus propios medicamentos, llamar por teléfono y salir a lugares cercanos. Las variables de AAVD incluyeron preguntas para evaluación de la movilidad del tren inferior (caminar y subir escalas), para movilidad del tren superior (levantar los brazos por sobre el nivel de la cabeza) y para evaluación de fuerza muscular de extremidades superiores (capacidad de acarrear o levantar paquetes) e inferiores (capacidad para sentarse y pararse desde una silla y subir escalas) ${ }^{17}$. Se incluyeron dentro de las AAVD algunas de las actividades propuestas por Nagi ${ }^{18}$ como agacharse, levantar $5 \mathrm{~kg}$ de peso y recoger una moneda desde una superficie lisa, las cuales fueron observadas y registradas.

Se determinó masa magra, masa grasa y masa ósea por absorciometría dual de rayos $\mathrm{X}$ $(D E X A)^{19}$. El peso fue registrado en una balanza de plataforma SECA (Madison, WI, U.S.A.) graduada a $0,1 \mathrm{~kg}$ y la talla fue medida con precisión de $0,5 \mathrm{~cm}$, utilizando estadiómetro adosado a la balanza, con el sujeto descalzo. La altura de rodilla fue medida con precisión de $0,1 \mathrm{~cm}$, con un «cáliper» de hoja ancha, con el muslo y la pierna en un ángulo de $90^{\circ}$ y el sujeto sentado. La circunferencia de cintura fue medida con el sujeto de pie, inmediatamente por encima de la cresta ilíaca, con una huincha SECA ad hoc. Las circunferencias de brazo y de pantorrilla fueron medidas con huincha flexible y precisión de $0,1 \mathrm{~cm}$. La primera en el punto medio del brazo, con éste colgando al costado del cuerpo y la segunda en la parte media de la zona carnosa de la pantorrilla. Las variables de peso y talla se usaron para calcular el índice de masa corporal y determinar el estado nutricional utilizando las categorías de IMC según el criterio $\mathrm{OMS}^{20}$. La circunferencia de cintura se categorizó utilizando los puntos de corte de riesgo $>102 \mathrm{~cm}$ en hombres y $>88 \mathrm{~cm}$ en mujeres ${ }^{21}$.

Se realizaron mediciones de fuerza de agarre de la mano con dinamómetro (Hand Dynamometer T18; Country Technology) utilizando la mano dominante y registrando la mayor de 2 mediciones.

Estadística. Las variables antropométricas y de composición corporal se describen como promedio, desviación estándar e intervalos de confianza. Se efectuó análisis de correlación simple entre indicadores antropométricos y de composición corporal. Se evaluó mediante test $\mathrm{chi}^{2}$ la asociación de indicadores antropométricos y de composición corporal con grupos con y sin limitación funcional. Se calcularon razones de prevalencia para la asociación entre limitación funcional y estado nutricional según IMC. La dinamometría se categorizó de acuerdo a la distribución percentilar de la dinamometría obtenida en la muestra total del estudio SABE a la cual pertenece esta submuestra. El percentil 25 correspondió a $27 \mathrm{~kg}$ en hombres y $15 \mathrm{~kg}$ en mujeres, el percentil 50 a $32,8 \mathrm{~kg}$ en hombres y $18,6 \mathrm{~kg}$ en mujeres y el percentil 75 a 39 $\mathrm{kg}$ en hombres $22 \mathrm{~kg}$ en mujeres. Se efectuaron regresiones logísticas múltiples para estudiar la asociación de funcionalidad y movilidad con IMC, dinamometría e indicadores antropométricos. Los análisis se realizaron con el programa Stata 8.0.

\section{Resultados}

La edad promedio fue de 73,9 años (DS 6,1; rango 65-91) en los hombres y 74,4 (DS 6,6; rango 6595) años en las mujeres.

Las características antropométricas de la muestra, según sexo, se describen en la Tabla 2. Los valores mayores correspondieron a los hombres, salvo en IMC, mayor en mujeres. Las diferencias fueron significativas en todas las variables, excepto para la circunferencia de brazo.

La composición corporal por sexo y grupos de edad, se describe en la Tabla 3. La masa magra y 
Tabla 2. Características antropométricas del grupo de adultos mayores en estudio según sexo

\begin{tabular}{|lllllll|}
\hline Variables & \multicolumn{2}{l}{ Hombres N =139 } & & Mujeres N =238 & \\
antropométricas & Promedio \pm DS & Min-máx & IC (95\%) & Promedio \pm DS & Min-máx & IC (95\%) \\
\hline Peso kg* & $73,6 \pm 12,4$ & $38,3-109,2$ & $71,5-75,7$ & $63,0 \pm 12,0$ & $36,5-109,1$ & $61,5-64,5$ \\
Talla cm* & $163,8 \pm 7,6$ & $132,0-183,5$ & $162,5-165,1$ & $148,5 \pm 6,3$ & $133,0-168,0$ & $147,7-149,3$ \\
IMC* kg/m² & $27,4 \pm 3,9$ & $16,3-36,4$ & $26,7-28,0$ & $28,5 \pm 4,7$ & $17,2-44,9$ & $27,9-29,1$ \\
Circ cintura cm* & $99,8 \pm 10,1$ & $66,0-123,5$ & $98,0-101,5$ & $94,3 \pm 11,6$ & $68,8-137,0$ & $92,8-95,7$ \\
Circ brazo cm & $30,0 \pm 3,3$ & $18,9-38,8$ & $29,4-30,5$ & $29,8 \pm 3,9$ & $17,5-40,0$ & $29,4-30,3$ \\
Circ pantorilla* & $35,3 \pm 3,7$ & $16,0-43,5$ & $34,7-35,9$ & $33,3 \pm 3,6$ & $23,0-42,5$ & $32,9-33,8$ \\
Altura rodilla cm* & $51,8 \pm 2,8$ & $41,5-59,0$ & $51,3-52,2$ & $46,8 \pm 2,7$ & $40,5-56,0$ & $46,5-47,2$ \\
Pliegue tríceps mm* & $19,2 \pm 9,3$ & $4,6-47,0$ & $17,6-20,7$ & $24,8 \pm 7,0$ & $4,3-40,3$ & $24,4-26,2$ \\
Dinamometría kg* & $29,6 \pm 8,9$ & $10,0-48,0$ & $28,1-31,1$ & $15,9 \pm 7,7$ & $0,2-32,0$ & $15,0-16,9$ \\
\hline
\end{tabular}

t test, ${ }^{*} p<0,02$.

Tabla 3. Composición corporal del grupo de estudio, según sexo y grandes grupos de edad

\begin{tabular}{|cllllll|}
\hline & \multicolumn{2}{c}{ Masa magra (kg) } & \multicolumn{2}{c|}{ Masa grasa (kg) } & \multicolumn{2}{c|}{ Masa ósea (kg) } \\
& Promedio \pm DS & IC (95\%) & Promedio \pm DS & IC (95\%) & Promedio \pm DS & IC (95\%) \\
\hline $\begin{array}{c}\text { Hombres } \\
<75 \text { a }\end{array}$ & $54,06 \pm 8,86^{* *}$ & $49,3-52,1$ & $24,91 \pm 7,61^{* *}$ & $22,4-26,1$ & $2,73 \pm 0,41$ & $2,6-2,8$ \\
$\geq 75$ a & $49,79 \pm 7,78^{* *}$ & $44,9-48,3$ & $21,62 \pm 6,88^{* *}$ & $19,8-23,5$ & $2,60 \pm 0,41$ & $2,5-2,7$ \\
Total & $48,9 \pm 6,4^{*}$ & $47,8-50,0$ & $22,9 \pm 7,5^{*}$ & $21,8-24,4$ & $2,6 \pm 0,4^{*}$ & $2,6-2,7$ \\
Mujeres & & & & & & \\
$\quad<75$ a & $37,91 \pm 5,82^{* *}$ & $34,8-36,7$ & $27,77 \pm 7,91$ & $26,1-29,1$ & $1,92 \pm 0,36^{* *}$ & $1,9-2,0$ \\
$\geq 75$ a & $35,9 \pm 6,12^{* *}$ & $32,8-34,7$ & $25,85 \pm 7,98$ & $23,4-26,8$ & $1,75 \pm 0,35^{* *}$ & $1,7-1,8$ \\
Total & $34,9 \pm 5,2^{*}$ & $34,1-35,5$ & $26,3 \pm 8,2^{*}$ & $25,3-27,3$ & $1,8 \pm 0,4^{*}$ & $1,8-1,9$ \\
\hline
\end{tabular}

t test ${ }^{*} \mathrm{p} \leq 0,0001$. **p $<0,02$.

la masa ósea fueron significativamente mayores en hombres y la masa grasa fue mayor en las mujeres $(p<0,0001)$. Se observó una significativa disminución de masa magra en los grupos mayores de 75 años. El IMC presentó muy alta correlación con la masa grasa en hombres $(r=0,87)$ y en mujeres $(r=0,91)$. Asimismo, se observó alta correlación entre IMC y masa magra en ambos sexos (hombres $r=0,55$; mujeres $r=0,63$ ).

La frecuencia de obesidad fue alta, mayor en mujeres $(35,7 \%)$ que en hombres $(25,2 \%)$ y la de bajo peso fue muy baja, tanto en hombres $(1,3 \%)$ como en mujeres $(2,2 \%)$.

Se observó mayor frecuencia de limitación funcional $(p<0,01)$ en mujeres $(63,7 \%)$ que en hombres (37,5\%). En el grupo $<75$ años, las frecuencias observadas fueron $30,8 \%$ en hombres y $48,9 \%$ en mujeres $(p<0,01)$ y en el grupo $\geq 75$ años, las frecuencias fueron $46,6 \%$ en hombres y $82,7 \%$ en mujeres ( $p<0,01)$. En el grupo de mayores de 75 años, la proporción de limitados en 20 más actividades fue muy superior a la del grupo de menores de 75 años en AVD, AIVD y AAVD, en ambos sexos (Tabla 4).

No se observó asociación entre limitación funcional y categorías de estado nutricional, ni entre niveles de riesgo de circunferencia de cintura y limitación funcional; aunque las razones de prevalencia de limitación funcional fueron menores en los grupos con sobrepeso y obesidad, 
Tabla 4. Limitación funcional en AVD , AIVD y AAVD en hombres y mujeres según grandes grupos de edad

\begin{tabular}{|clrrrrrr|}
\hline \multirow{2}{*}{$\begin{array}{c}\text { Número de } \\
\text { limitaciones }\end{array}$} & \multicolumn{2}{c}{ AVD \% } & \multicolumn{2}{c}{ AIVD \% } & \multicolumn{2}{c|}{ AAVD \% } \\
& & \multicolumn{2}{c}{ Edad (años) } & \multicolumn{2}{c|}{ Edad (años) } & \multicolumn{2}{c|}{ Edad (años) } \\
& & $<75$ & $\geq 75$ & $<75$ & $\geq 75$ & $<75$ & $\geq 75$ \\
\hline 0 & Hombres & 91,5 & 80,4 & 73,2 & 60,4 & 66,2 & 55,2 \\
& Mujeres & 87,8 & 60,4 & 59,8 & 22,4 & 50,5 & 21,6 \\
1 & Hombres & 2,8 & 7,1 & 12,7 & 11,5 & 14,1 & 15,5 \\
& Mujeres & 7,5 & 11,5 & 26,2 & 32,6 & 22,4 & 23,7 \\
$\geq 2$ & Hombres & 5,7 & 12,5 & 14,1 & 28,1 & 19,7 & 29,3 \\
& Mujeres & 4,8 & 28,1 & 14,0 & 45,0 & 27,1 & 54,7 \\
\hline
\end{tabular}

Tabla 5. Frecuencia de limitación funcional según estado nutricional y categorías de riesgo de circunferencia de cintura en hombres y mujeres

\begin{tabular}{|c|c|c|c|c|c|c|}
\hline & $\begin{array}{l}\text { Limitados } \\
\quad \mathrm{N}(\%)\end{array}$ & $\begin{array}{c}\text { Hombres } \\
\text { Sin limitación } \\
\mathrm{N}(\%)\end{array}$ & $\mathrm{RP} *$ & $\begin{array}{l}\text { Limitados } \\
\quad \mathrm{N}(\%)\end{array}$ & $\begin{array}{c}\text { Mujeres } \\
\text { Sin limitación } \\
\mathrm{N}(\%)\end{array}$ & $\mathrm{RP} *$ \\
\hline \multicolumn{7}{|l|}{ IMC $\left(\mathrm{kg} / \mathrm{m}^{2}\right)$} \\
\hline$<24,9$ & $14(48,3)$ & $15(51,7)$ & 1 & $41(70,7)$ & $17(29,3)$ & 1 \\
\hline $25-29,9$ & $27(37,0)$ & $46(63,0)$ & 0,77 & $51 \quad(54,3)$ & $43(45,7)$ & 0,76 \\
\hline$\geq 30$ & $10(29,4)$ & $24(70,6)$ & 0,61 & $59(69,4)$ & $26(30,6)$ & 0,98 \\
\hline \multicolumn{7}{|l|}{ Cintura (cm) } \\
\hline$H<94 M<80$ & $13(38,2)$ & $21(61,8)$ & 1 & $17(68,0)$ & $8(32,0)$ & 1 \\
\hline H 94-102 M 80-88 & $21(42,0)$ & $29(58,0)$ & 1,10 & $29(53,7)$ & $25(46,3)$ & 0,79 \\
\hline $\mathrm{H}>102 \mathrm{M}>88$ & $17(31,5)$ & $37(68,5)$ & 0,82 & $105(65,6)$ & $55(34,4)$ & 0,96 \\
\hline
\end{tabular}

*Razón de prevalencia de limitación funcional.

no alcanzaron significación estadística (Tabla 5). Tampoco se observó asociación entre limitaciones funcionales y porcentaje de grasa corporal (no mostrado).

En la Tabla 6, se presenta la asociación de limitación funcional con dinamometría. Tanto hombres como mujeres en el primer cuartil de dinamometría, presentaron significativo mayor riesgo de limitación funcional para caminar 8 cuadras, para subir escaleras y para extender los brazos sobre nivel de hombros. En mujeres también fue significativa la diferencia para transportar $5 \mathrm{~kg}$.

Para ajustar la relación de dinamometría con limitación funcional por edad y por otras variables antropométricas, se efectuaron análisis de regresión múltiple en el cual se incluyeron: dinamome- tría, edad, IMC, altura de rodilla y circunferencias de cintura, pantorrilla y brazo. En ambos sexos se mantuvo la asociación significativa entre dinamometría y limitación funcional, agregándose en mujeres mayor riesgo de limitación funcional a mayor edad y mayor IMC (Tabla 7). El resto de las variables antropométricas estudiadas no presentó asociación con limitación funcional.

\section{DISCUSIÓN}

La discapacidad se define como la disminución de la habilidad para efectuar tareas y actividades específicas que son esenciales para el cuidado personal y la autonomía ${ }^{22}$. Las limitaciones fun- 


\section{Tabla 6. Riesgo de limitación de movilidad para sujetos en el primer cuartil de dinamometría* en relación a cuartiles II, III y IV}

\begin{tabular}{|lccrrrr|}
\hline Limitación para: & OR & Hombres & & \multicolumn{3}{c|}{ Mujeres } \\
\hline Subir escaleras, un piso & 2,86 & $1,16-7,08$ & 0,011 & 2,69 & $1,54-4,70$ & 0,001 \\
Caminar 8 cuadras & 4,97 & $1,86-13,83$ & $<0,001$ & 2,89 & $1,64-5,10$ & $<0,001$ \\
Agacharse a recoger lápiz & 1,82 & $0,15-21,58$ & 0,630 & 2,46 & $0,77-7,21$ & 0,068 \\
Extender brazos sobre nivel de hombros & 2,50 & $1,05-5,99$ & 0,022 & 2,32 & $1,33-4,07$ & 0,002 \\
Transportar 5 kg & 3,71 & $0,04-29,7$ & 0,333 & 5,75 & $2,36-14,08$ & $<0,001$ \\
Limitación 2 o mas ítem de movilidad & 9,56 & $2,87-32,73$ & $<0,001$ & 12,29 & $5,03-33,95$ & $<0,001$ \\
\hline
\end{tabular}

*hombres $<27 \mathrm{~kg}$; mujeres $<15 \mathrm{~kg}$.

Tabla 7. Riesgo de limitación funcional para variables antropométricas como variables independientes

\begin{tabular}{|lccccrr|}
\hline \multirow{2}{*}{ Limitación funcional } & \multicolumn{3}{c}{ Hombres $\left(\mathrm{n}=136 ; \mathrm{r}^{2}=0,12\right)$} & \multicolumn{3}{c|}{ Mujeres $\left(\mathrm{n}=236 ; \mathrm{r}^{2}=0,11\right)$} \\
& OR & $\mathrm{p}$ & $95 \% \mathrm{IC}$ & OR & $\mathrm{p}$ & $95 \% \mathrm{IC}$ \\
\hline Dinamometría $(\mathrm{kg})$ & 0,93 & 0,002 & $0,867-0,968$ & 0,958 & 0,039 & $0,917-0,998$ \\
Edad & 1,04 & 0,122 & $0,975-1,117$ & 1,098 & $<0,001$ & $1,043-1,155$ \\
IMC $\left(\mathrm{kg} / \mathrm{m}^{2}\right)$ & 0,95 & 0,398 & $0,830-1,077$ & 1,105 & 0,039 & $1,005-1,214$ \\
\hline
\end{tabular}

cionales constituyen el componente principal de los modelos que explican la génesis de la discapacidad, ya que se consideran el estado de transición entre las etapas de deficiencia y de discapacidad $^{23}$. Pope y Tarlov describen una tasa desproporcionadamente alta de discapacidad en los más viejos, en las minorías y en los más pobres $^{22}$, lo cual ha sido reiterado en estudios posteriores ${ }^{24,25}$ y coincide con nuestros resultados en que la prevalencia de limitación funcional fue muy alta, mayor en los más viejos y en las mujeres.

En el presente estudio no encontramos asociación de grasa corporal e IMC con limitación funcional. Se encontró alta frecuencia de obesidad, tanto en mujeres como en hombres y muy baja prevalencia de bajo peso. El IMC presentó muy alta asociación con la grasa corporal, pero no se observó asociación de estos indicadores con limitación funcional. Tampoco observamos asociación de circunferencia de cintura con limitación funcional ni movilidad. Estos resultados no concuerdan con hallazgos previos que demuestran asociación de grasa corporal y de obesidad con limitación funcional y con actividades de movilidad ${ }^{12,26}$. Al respecto, debe considerarse que en nuestro estudio el IMC también presentó alta correlación con la masa magra en ambos sexos. De acuerdo a los estudios de Baumgartner ${ }^{27}$, sólo los sujetos con obesidad sarcopénica presentan riesgo aumentado de limitación funcional, a diferencia de los individuos obesos sin sarcopenia, que presentan un riesgo similar al de los individuos normales.

En la búsqueda de indicadores antropométricos y funcionales que mejor se correlacionen con la funcionalidad, el principal hallazgo de este estudio fue la estrecha relación de la dinamometría con la funcionalidad y con la habilidad para efectuar actividades de movilidad. En los modelos de regresión múltiple que incluyeron edad y las variables antropométricas estudiadas, sólo la dinamometría mantuvo asociación significativa con funcionalidad en ambos sexos, agregándose la edad como factor de riesgo en las mujeres. Aunque se observó una buena correlación de la fuerza de agarre con la masa magra, la asociación de la fuerza de agarre con 
funcionalidad fue mayor que la explicada sólo por la diferencia en la masa muscular.

La masa y la fuerza muscular son importantes determinantes de la función física en adultos mayores. Diversos estudios han demostrado que existe una estrecha relación entre fuerza y masa muscular y que la combinación de sarcopenia y pérdida de fuerza muscular contribuye en forma significativa a la declinación de la habilidad funcional e independencia que se produce con el envejecimiento $^{28}$. También se ha observado que la disminución de la fuerza muscular es predictiva de declinación funcional futura, incidencia de discapacidad y mayor riesgo de mortalidad $^{13,29}$. Nuestro estudio confirma que la pérdida de fuerza muscular no se explica solamente por la disminución de la masa muscular. Este hallazgo coincide con estudios efectuados que demuestran que la disminución del volumen muscular explica sólo la mitad de la disminución de la fuerza muscular que se produce con el envejecimiento ${ }^{30}$.

La disminución, tanto de la masa como de la fuerza muscular que se produce con el envejecimiento, está bien documentada $7,31,32$. Estudios transversales han mostrado que la disminución de la masa muscular se asocia con un pobre rendimiento funcional ${ }^{33}$ y con autorreporte de discapacidad $^{11,34}$. En adición a la disminución del volumen muscular, la composición del músculo cambia $^{35-37}$, produciéndose una creciente infiltración de grasa, característica importante de la sarcopenia. Estudios recientes ${ }^{33,38}$ han mostrado una asociación entre la cantidad de infiltración grasa en el músculo y la fuerza muscular y movilidad ${ }^{39}$. En suma, la evidencia actual apunta a que la disminución de la masa muscular, la alta infiltración grasa en el músculo y la menor fuerza muscular se asocian con riesgo mayor de pérdida de movilidad en ancianos.

Este estudio demuestra que la función muscular es más importante que el tamaño de la masa muscular y valida la dinamometría de mano como

\section{REFERENCIAS}

1. Albala C, Vio F, Kain J, Uauy R. Nutrition Transition in Latin America: The Case of Chile. Nutr Reviews 2001; 56: 170-6. un indicador de funcionalidad. Este hallazgo concuerda con estudios que han demostrado que la dinamometría de mano entrega estimaciones de riesgo de mortalidad similares a las de fuerza de cuádriceps y que esta asociación es independiente del IMC 29,40 .

Una debilidad de este estudio es su carácter transversal, ya que se trata de grupos que pertenecen a cohortes diferentes (nacidos entre 1910 y 1940), que crecieron en condiciones muy disímiles, lo que eventualmente puede introducir sesgos en su comparación, pero su fortaleza reside en el gran número de sujetos estudiados (incluyendo 79 mayores de 80 años), evaluados con una batería completa de mediciones antropométricas, composición corporal medida con DEXA y observación directa de actividades de movilidad.

La etapa de limitación funcional se considera un estado de alto riesgo de discapacidad ${ }^{41}$, pero también una etapa en que se puede obtener el máximo beneficio de intervenciones ${ }^{22}$. La masa muscular, fuerza muscular y la infiltración grasa en el músculo, pueden ser modificadas con intervenciones conductuales y farmacológicas en personas mayores ${ }^{42,43}$. El aumento de la fuerza muscular observada en estudios de intervención se obtiene, incluso, con pequeños incrementos de masa ma$\mathrm{gra}^{44}$. Intervenciones efectuadas en la comunidad han demostrado aumento de la fuerza muscular y de la capacidad de marcha con un programa de ejercicios de resistencia progresiva ${ }^{10,45}$.

La mayoría de las discapacidades son prevenibles a través de intervenciones oportunas, con la consiguiente mejoría en la calidad de vida y gran ahorro en los costos resultantes de la dependencia, pérdida de productividad y cuidado de la salud.

Este estudio valida un indicador de fácil uso, rápido y de bajo costo en la evaluación geriátrica. Su uso ayudará a optimizar la identificación de sujetos en riesgo y la evaluación de estrategias de intervención enfocadas hacia la mantención de la funcionalidad en adultos mayores.

2. BerRY E. Chronic Disease: How can Nutrition Moderate the Effects? Nutrition Reviews 1994; 52 (suppl 2): S28-S30.

3. Lawrence RH, Jette AM. Disentangling the Disablement Process. J Gerontol B Psychol Sci Soc Sci 1996; 51: S173-182. 
4. Reuben D, Rubenstein L, Hirsch S. Value of Functional Status as a Predictor of Mortality: Results of a Prospective Study. Am J Med 1992; 93: 663-9.

5. Verbrugge LM, Jette AM. The Disablement Process. Soc Sci Med 1994; 38: 1-14.

6. Roubenoff R. Sarcopenia: Effects on Body Composition and Function. J Gerontol A Biol Sci Med Sci 2003; 58: 1012-17.

7. Evans W. What is Sarcopenia?J Gerontol 1995; 50a (Special Issue): 5-8.

8. Evans W. Functional and Metabolic Consequences of Sarcopenia. J Nutr 1997; 127: 998S-1003S.

9. Baumgartner RN, Koehler KM, Galagher D, Romero L, Heymsfield SB, Ross RR et al. Epidemiology of Sarcopenia Among Elderly in New Mexico. Am J Epidemiol 1998; 147: 755-63.

10. Sternfeld B, Ngo L, Satariano WA, Tager iB. Associations of Body Composition with Physical Performance and Self-Reported Functional Limitation in Elderly Men and Women. Am J Epidemiol 2002; 156: 110-21.

11. Rantanen T, Harris T, Leveilie SG. Muscle Strength and Body Mass Index as Long Term Predictors of Mortality in Initially Healthy Men. J Gerontol A Biol Sci Med Sci 2000; 55: M168-73.

12. Davidson KK, Ford ES, Cogswell ME, Dietz WH. Percentage of Body Fat and Body Mass Index are Associated with Mobility Limitations in People Aged 70 and Older from NHANES III. J Am Geriatr Soc 2002; 50: 1802-9.

13. Bunout D, Barrera $G$, Avendaño M, De la Maza MP, Gattas V, Leiva L et al. Results of a Community-Based Weight-Bearing Resistance Training Program for Healthy Chilean Elderly Subjects. Age \& Ageing 2005; 34: 80-3.

14. Albala C, Lebrão ML, León Díaz EM, Ham-Chande R, Hennis AJ, PaLoni A et al. Encuesta Salud, Bienestar y Envejecimiento (SABE): Metodología de la Encuesta y Perfil de la Población Estudiada. Rev Panam Salud Pública 2005; 17: 307-22.

15. MC Dowel I, Newell C. Physical Disability and Handicap. Measuring Health, A Guide for Rating Scales and Questionnaires. $2^{\text {nd }}$ edition, New York Oxford University Press 1996, pp-121.

16. Albala C, Lera L, García C, Arroyo P, Marín PP, Bunout D. Searching a Common Definition for Functional Limitation in Latin America. The Gerontologist 2004; 44 (Special Issue I): 550s.

17. Rosow I, BresLau MA. Guttman Health Scale for the Aged. J Gerontol 1966; 21: 556-9.
18. NAGI SZ. An Epidemiology of Disability among Adults in the United States. Milbank Memorial Fund 1976; 54: 439-68.

19. Salamone LM, Fuerst T, Visser M, Kern M, Lang T, DockReLL M ET AL. Measurement of Fat Mass Using DEXA: a Validation Study in Elderly Adults. J Appl Physiol 2000; 89: 345-52.

20. World Health Organization, WHO. Obesity: preventing and managing the global epidemic. Report of a WHO consultation on obesity, Geneva 1997. WHO/NUT/NCD/98.1.

21. Lfan MEJ, Han TS, MorRISON CE. Waist Circumference as a Measure for Indicating Need for Weight Management. BMJ 1995; 311: 158-61.

22. Pope AM, Tarlov AR. Disability in America: Toward a National Agenda for Prevention. National Academy Press. Washington, DC 1991 Accessed at http:// darwin.nap.edu/books/0309043786/html

23. Simonsick EM, Kasper JD, GuRALniK JM, BandeENRoche K, FerRucci L, Hirsch R et al for the WHAS Research Group. Severity of Upper and Lower Extremity Functional Limitation: Scale Development and Validation with Self-Report and Performance-Based Measures of Physical Function. J Gerontol B Psychol Sci Soc Sci 2001; 56: S10-S19.

24. Tucker KL, Falcon LM, Bianchi LA, CAcho E, BermúdeZ O. Self Reported Prevalence and Health Correlates of Functional Limitation among Massachusetts Elderly Puerto Ricans, Dominicans and a non-Hispanic White Neighborhood Comparison Group. J Gerontol A Biol Sci Med Sci 2000; 55: M90-M97.

25. Kuh D, Bassey EJ, Butterworth S, Hardy R, Wadsworth M, The Musculoskeletal Study Team. Grip Strength, Postural Control, and Functional Leg Power in a Representative Cohort of British Men and Women: Associations with Physical Activity, Health Status and Socioeconomic Conditions. J Gerontol A Biol Sci Med Sci 2005; 60: 224-31.

26. Zoico E, Di Francesco V, Guralnik JM, Mazzali G, Bortolani A, Guariento S et al. Physical Disability and Muscular Strength in Relation to Obesity and Different Body Composition Indexes in a Sample of Healthy Elderly Women. Int J Obes 2004; 28: 234-41.

27. Baumgartner R, Wayne SJ, Waters DL, Janssen I, Galagher D, Moriey JE. Sarcopenic Obesity Predicts Instrumental Activities of Daily Living Disability in the Elderly. Obes Res 2004; 12: 1995-2004.

28. DohERTY TJ. Invited Review: Aging and Sarcopenia. J Appl Physiol 2003; 95: 1717-27. 
29. Newman A, Kupelian V, Visser M, Simonsick E, GooDPASTER BH, KRTTCHEVSKY SB ET AL. Strength but not Muscle Mass is Associated with Mortality in the Health, Aging and Body Composition Study Cohort. J Gerontol A Biol Sci Med Sci 2006; 61: 72-7.

30. Thom JM, Morse C, Birch K, Narici M. Triceps Surae Muscle Power, Volume and Quality in Older versus Younger Healthy Man. J Gerontol A Biol Sci Med Sci 2005; 60: 111-7.

31. Landers KA, Hunter GR, Wetzstein CJ, Bamman MM, WeINSIER RL. The Interrelationship among Muscle Mass, Strength, and the Ability to Perform Physical Tasks of Daily Living in Younger and Older Women. J Gerontol 2001; 56: B443-B448.

32. Frontera WR, Hughes VA, Fielding RA, Fiatarone MA, Evans WJ, Roubenoff R. Aging of Skeletal Muscle: a 12-yr Longitudinal Study. J Appl Physiol 2000; 88: 1321-6.

33. Visser M, Kritchevsky SB, Goodpaster BH, Newman AB, Nevitt M, Stamm E et al. Leg Muscle Mass and Composition in Relation to Lower Extremity Performance in Men and Women aged 70 to 79 : the Health, Aging and Body Composition Study. J Am Geriatr Soc 2002; 50: 897-904.

34. JaNSSEN I, HeYMSFEeID SB, Ross R. Low Relative Skeletal Muscle Mass (Sarcopenia) in Older Persons is Associated with Functional Impaiment and Physical Disability. J Am Geriatr Soc 2002; 50: 889-96.

35. Borkan GA, Hults DE, Gerzof SG, Robbins AH, SilberT CK. Age Changes in Body Composition Revealed by Computed Tomography. J Gerontol 1983; 38: 673-7.

36. Forsberg AM, Nilsson E, Werneman J, Bergstrom J, Hultman E. Muscle Composition in Relation to Age and Sex. Clin Science 1991; 81: 249-56.

37. Overend TJ, Cunningham DA, Paterson DH, Lefcoe MS. Thigh Composition in Young and Elderly Men Determined by Computed Tomography. Clin Physiol 1992; 12: 629-40.

38. Goodpaster BH, Carlson CL, Visser M, Keluey DE, Scherzinger A, Harris TB et al. The Association between Skeletal Muscle Composition and Strength in the Elderly: the Health ABC Study. J Appl Physiol 2001; 90: 2157-65.

39. Visser M, Goodpaster BH, Kritchevsky SB, Newman AB, Nevitt M, Rubin SM et al, For The Health Abc Study. Muscle Mass, Muscle Strength, and Muscle Fat Infiltration as Predictors of Incident Mobility Limitations in Well-Functioning Older Persons. J Gerontol A Biol Sci Med Sci 2005; 60: 324-33.

40. Rantanen T, Guralnik JM, Ferrucci L, Penninx BW, LeVeile S, Sipila S et al. Coimpairments as predictors of severe walking disability in older women. J Am Geriatr Soc 2001; 49: 21-7.

41. Guralnik JM, Ferrucci L, Pieper CF, Leveide SG, Markides KS, OstiR GV ET AL. Lower Extremity Function and Subsequent Disability: Consistency Across Studies, Predictive Models, and Value of Gait Speed Alone Compared With the Short Physical Performance Battery. J Gerontol A Biol Sci Med Sci 2000; 55: M221-M231.

42. Phiшips W, Hazeldene R. Strength and Muscle Mass Changes in Elderly Men Following Maximal Isokinetic Training. Gerontology 1996; 42: 114-20.

43. Ryan AS, Nickias BJ, Berman DM, Dennis KE. Dietary Restriction and Walking Reduce Fat Deposition in the Mid Thigh in Obese Older Women. Am J Clin Nutr 2000; 72: 708-13.

44. Morganti CM, Nelson ME, Fiatarone MA, Dalia GE, Economos CD, CRAWFord BM ET AL. Strength Improvements with 1 year of Progressive Resistance Training in Older Women. Med Sci Sports Exerc 1995; 27: 906-12.

45. Gill TM, Baker Di, Gottschalk M, Peduzzi PN, Alore H, Byers A. A Program to Prevent Functional Decline in Physically Frail, Elderly Persons Who Live at Home. N Engl J Med 2002; 347: 1068-74.

46. Haines TP, Bennel KL, Osborne RH, Hill KD. Effectiveness of Targeted Falls Prevention Program in Subacute Hospital Setting: Randomized Controlled Trial. BMJ 2004; 328: 676-82. 